\title{
NOTE ON STRONG SOLUTIONS OF A STOCHASTIC INCLUSION
}

\author{
JERZY MOTYL \\ Higher College of Engineering \\ Institute of Mathematics \\ Podgórna 50, 65-246 Zielona Góra \\ Poland
}

(Received February, 1995; Revised March, 1995)

\begin{abstract}
Two different definitions of strong solutions of a stochastic integral set-valued equation are discussed. A selection property of a set-valued stochastic integral is given.
\end{abstract}

Key words: Semimartingale, Predictable Set-Valued Function, $M$-integrable Selector, Hausdorff Metric.

AMS (MOS) subject classifications:93E03, 93C30.

\section{Introduction}

In the theory of stochastic equations the definition of their solutions is quite natural. A process $x$ is a solution of the equation

$$
x_{t}=\int_{0}^{t} f_{\tau}(x) d M_{\tau}, \quad 0 \leq t<\infty
$$

if the above is satisfied for all $t$.

In the set-valued approach there are two possibilities for defining a solution of a stochastic inclusion.

Let $F$ be a set-valued predictable process and let the following stochastic inclusion be given:

$$
x_{t} \in \int_{0}^{t} F_{\tau}(x) d M_{\tau}, \quad 0 \leq t<\infty
$$

(for required definitions see the next section).

Definition A: A process $x$ is a solution of problem (2) if it satisfies

$$
x_{t}-x_{s} \in \int_{s}^{t} F_{\tau}(x) d M_{\tau}
$$

for all $0 \leq s<t<\infty$.

Definition B: A process $x$ is a solution of problem (2) if there exists an $M$-integrable selector $f$ of $F(x)$ such that 
for all $t, 0 \leq t<\infty$.

$$
x_{t}=\int_{0}^{t} f_{\tau} d M_{\tau}
$$

Definition $\mathbf{A}$ is more natural because of its similarity to a single-valued case. In stochastic set-valued investigations the two definitions have been used. In $[1,10,11]$ the solutions were investigated in the sense of $\mathbf{B}$, while in $[7,8]$ they were investigated in the sense of $\mathbf{A}$. Avgerinos and Papageorgiou in [3] used a combination of these definitions. They investigated a random inclusion of the type

$$
\dot{x}(\omega, t) \in A(\omega) x(\omega, t)+F(\omega, t, x(\omega, t))
$$

and as a solution they meant a process satisfying the inclusion

$$
\dot{x}(\omega, t) \in A(\omega) x(\omega, t)+f(\omega)(t)
$$

for $f(\omega)$ being a selection of $F(\omega, \cdot, x(\omega, \cdot))$.

It is well known that, in the ordinary differential inclusion case, these two concepts of solutions coincide only for convex-valued set-valued functions (see e.g., Integral Representation Property in [2, p. 99]). The same is true for a stochastic inclusion with a Wiener process ([7, Th. $4.1])$, but it is an open problem for the semimartingale case. It is clear that if $x$ is a solution of problem (2) in the sense of definition $\mathbf{B}$, it is also a solution in the sense of $\mathbf{A}$. The purpose of this paper is to prove the converse, and this requires some selection-type theorem.

\section{Preliminaries}

Throughout the paper $\left.\left(\Omega, \mathscr{F}_{,}, \mathscr{F}_{t}\right\}_{t \geq 0}, P\right)$ denotes a complete filtered probability space satisfying the usual hypothesis: $(i) \mathscr{F}_{0}$ contains all $P$-null sets of $\mathscr{F},(i i) \mathscr{F}_{t}=\bigcap_{u>t} \mathscr{F}_{u}$, for all $t, 0 \leq$ $t<\infty$; This means that a filtration $\left\{\mathscr{F}_{t}\right\}_{t \geq 0}$ is right continuous. By a stochastic process $x$ on $(\Omega, \mathcal{F}, P)$ we mean a collection $\left(x_{t}\right)_{t \geq 0}$ of $n$-dimensional random variables $x_{t}: \Omega \rightarrow \mathbb{R}^{n}, t \geq 0$. The process $x$ is said to be adapted if $x_{t}$ belongs to $\mathscr{F}_{t}$ (which means it is $\mathscr{F}_{t}$-measurable) for each $t \geq 0$. A stochastic process $x$ is called cádlág if it a.s. has sample paths which are right continuous, with left limits. Similarly, a stochastic process $x$ is said to be cáglád if it a.s. has sample paths which are left continuous, with right limits. The family of all adapted cádlág (cáglád) processes is denoted by $D[L]$.

Let $\mathscr{P}\left(\mathscr{F}_{t}\right)$ denote the smallest $\sigma$-algebra on $\mathbb{R}_{+} \times \Omega$ with respect to which every cáglád adapted process is measurable in $(t, \omega)$, i.e. $\mathscr{P}\left(\mathscr{F}_{t}\right)=\sigma(L)$. A stochastic process $x$ is said to be predictable if $x$ is $\mathscr{P}\left(\mathscr{F}_{t}\right)$-measurable. The family of all such processes is denoted by $\mathscr{P}$. One has $\mathscr{P}\left(\mathscr{F}_{t}\right) \subset \beta_{+} \otimes \mathscr{F}$, where $\beta_{+}$denotes the Borel $\sigma$-algebra on $\mathbb{R}_{+}$.

Denote $X^{2}=\left\{x \in \mathscr{P}:\|x\|_{S^{2}}<\infty\right\}$, where $\|x\|_{S^{2}}=\left\|\sup _{t \geq 0}\left|x_{t}\right|\right\|_{L^{2}}$. It can be verified that $\left(X^{2},\|\cdot\|_{S^{2}}\right)$ is a Banach space (see e.g., $\left.[12,13]\right)$.

Let $\mathcal{H}$ [or $\mathcal{M}_{0}$ ] denote the set of all one-dimensional semimartingales [or vanishing at $t=0$ respectively]. Given $M \in \mathcal{M}$, let $M=N+A$ be a decomposition of $M$, where $N$ is a local martingale, $A$ denotes a process with path of finite variation on compacts and $[N, N]$ denotes the quadratic variation process of $N$. Define

$$
j_{2}(N, A)=\left\|[N, N]_{\infty}^{\frac{1}{2}}+\int_{0^{-}}^{\infty}\left|d A_{s}\right|\right\|_{L^{2}}
$$


and

$$
\|M\|_{J_{G}^{2}}=\inf _{M=N+A} j_{2}(N, A)
$$

where $\int_{0}^{t}\left|d A_{s}\right|=\int_{[0, t]}\left|d A_{s}\right|$ and the infimum is taken over all possible decompositions of $M$. Define ${ }^{0} \mathcal{G}^{2}=\left\{M \in \mathcal{M}^{[0}:\|M\|_{\mathcal{H G}^{2}}<\infty\right\}$. We also let $L(M)=\{H \in \mathscr{P}: H$ is integrable with respect to $M\}$ with a norm $\|H\|_{M}=\|H \cdot M\|_{\mathfrak{H}^{2}}$. Moreover, by $H \cdot M$ we denote $\int H_{\tau} d M_{\tau}$.

Let $\mathbb{R}^{n}$ be the $n$-dimensional Euclidean space and $C l\left(\mathbb{R}^{n}\right), \operatorname{Comp}\left(\mathbb{R}^{n}\right)$ and $\operatorname{Conv}\left(\mathbb{R}^{n}\right)$ denote spaces of all nonempty closed, compact, compact and convex, respectively, subsets of $\mathbb{R}^{n}$. Denote by $\operatorname{dist}(a, A)$ the distance between $a \in \mathbb{R}^{n}$ and $A \in C l\left(\mathbb{R}^{n}\right)$. We put $\bar{h}(A, B)=\sup _{a \in B} \operatorname{dist}(a, A)$, and $h(A, B)=\max \{\bar{h}(A, B), \bar{h}(B, A)\}$ for all $A, B \in C l\left(\mathbb{R}^{n}\right)$.

Consider a set-valued stochastic process $\mathfrak{B}_{0}=\left(\mathscr{R}_{t}\right)_{t}>0$ with values in $C l\left(\mathbb{R}^{n}\right)$, i.e. a family of $\mathscr{F}$-measurable set-valued mappings $\Re_{t}: \Omega \rightarrow C l\left(\mathbb{R}^{n}\right)$ for each $t \geq 0$. We call $\Re$ predictable if $\mathscr{R}$ is $\mathscr{P}\left(\mathscr{F}_{t}\right)$-measurable in the sense of set-valued functions.

Given a predictable set-valued process $\mathscr{B}_{0}=\left(\mathscr{R}_{t}\right)_{t \geq 0}$ and $M \in \mathcal{M}_{0}$, let

$$
\varphi_{M}\left(\mathscr{B}_{0}\right):=\left\{H \in L(M): H_{t} \in \mathscr{B}_{t} \text { for all } t\right\}
$$

A set $\varphi_{M}(\Re)$ is called a subtrajectory integral of $\mathscr{R}$.

A predictable set-valued process $\mathscr{R}$ is said to be integrable with respect to a semimartingale $M$ or, simply, $M$-integrable, if $\varphi_{M}(\Re)$ is a nonempty set. It follows immediately from the properties of stochastic integrals with respect to semimartingales (see Th. 3.2 of [6]) and Kuratowski and Ryll-Nardzewski measurable selection theorem (see e.g. [9]), that every $M$-integrably bounded and predictable set-valued stochastic process $\Re$ is $M$-integrable. Recall a set-valued stochastic process $\mathscr{R}_{0}=\left(\mathscr{R}_{t}\right)_{t \geq 0}$ is $M$-integrably bounded if there exists $m \in L(M) \cap X^{2}$ such that $h\left(\Re_{t},\{0\}\right) \leq m_{t}$ a.s., for each $t \geq 0$.

\section{Selection Properties of Integrals} formulas.

Convention: In this section we employ a notation $\int_{a}^{b} H d M$ instead of $\int_{a}^{b} H_{s} d M_{s}$ for clarity of
mulas.

Lemma 1: Let $M$ be a semimartingale in ${ }^{6}{ }^{2}$, let $x=\left(x_{t}\right)_{t>0}$ be a cádlág process and let a predictable set-valued process $\mathcal{G}$ be integrably bounded by a process $m=\left(m_{t}\right)_{t \geq 0}, m \in L(M)$. If $x_{t}-x_{s} \in c l L_{L}^{2} \int_{s}^{t} \mathrm{~g} d M$ for every $0 \leq s<t<\infty$, then for all stopping times $\alpha, \beta, 0 \leq \alpha<\beta<\infty$, there exists a sequence $\left(g^{n}\right) \subset c l_{L(M)}{ }_{M}(\mathfrak{g})$ such that

$$
\lim _{n \rightarrow \infty}\left\|\left(x_{\beta}-x_{\alpha}\right)-\int_{\alpha}^{\beta} g^{n} d M\right\|_{L^{2}}=0 .
$$

Proof: Let $\alpha_{n}=k \cdot 2^{-n}$ for $\omega$ such that $(k-1) 2^{-n} \leq \alpha(\omega)<k 2^{-n}$ and $\beta_{n}=k \cdot 2^{-n}$ for $\omega$ such that $(k-1) 2^{-n} \leq \beta(\omega)<k 2^{-n}$. Let $A_{k}^{n}=\left\{\omega: \alpha \geq k \cdot 2^{-n}\right\}$ and $B_{k}^{n}=\left\{\omega: \beta \geq k \cdot 2^{-n}\right\}$. Then we have

$$
\left[0, \alpha_{n}\right]=(\{0\} \times \Omega) \cup\left(\bigcup_{k=0}^{\infty}\left(k \cdot 2^{-n},(k+1) 2^{-n}\right] \times A_{k}^{n}\right)
$$


Now, for each $n=1,2, \ldots$ we obtain

$$
\left[0, \beta_{n}\right]=(\{0\} \times \Omega) \cup\left(\bigcup_{k=0}^{\infty}\left(k \cdot 2^{-n},(k+1) 2^{-n}\right] \times B_{k}^{n}\right) .
$$

$$
x_{\alpha_{n}}=x_{0}+\sum_{k=0}^{\infty} I_{A_{k}^{n}}\left(x_{(k+1) 2}-n-x_{k 2}-n\right)
$$

and

$$
x_{\beta_{n}}=x_{0}+\sum_{k=0}^{\infty} I_{B_{k}^{n}}\left(x_{(k+1) 2}-n-x_{k 2}-n\right) .
$$

Since $A_{k}^{n} \subset B_{k}^{n}$ then

$$
x_{\beta_{n}}-x_{\alpha_{n}}=\sum_{k=0}^{\infty} I_{B_{k}^{n} \backslash A_{k}^{n}\left(x_{(k+1) 2}-n-x_{k 2}-n\right) .}
$$

For every $k=0,1, \ldots$ and $n=1,2, \ldots$ we can select $g^{n, k} \in \varphi_{M}(\mathcal{G})$ such that

and put

$$
\left\|x_{(k+1) 2}-n-x_{k 2}-n-\int_{k 2}^{(k+1) 2-n} g^{n, k} d M\right\|_{L^{2}}<\epsilon /\left(3 \cdot 2^{k}\right)
$$

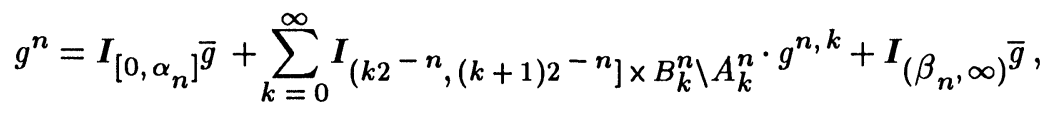

where $\bar{g} \in \varphi_{M}(\mathcal{G})$ is an arbitrary selector.

It is easy to see that $g^{n}$ belongs to $\left.c l_{L(M}\right)_{M}^{\varphi_{M}}(\mathcal{G})$ because of decomposability of $\varphi_{M}(\mathcal{G})$ and the Lebesgue Dominated Convergence Theorem. Moreover,

$$
\int_{\alpha_{n}}^{\beta_{n}} g^{n} d M=\sum_{k=0}^{\infty} I_{B_{k}^{n} \backslash A_{k 2}^{n}} \int_{k 2^{-n}}^{(k+1) 2-n} g^{n, k} d M
$$

Since $\left|g_{t}^{n}(\omega)\right| \leq m_{t}(\omega)$ for every $(t, \omega)$, we obtain

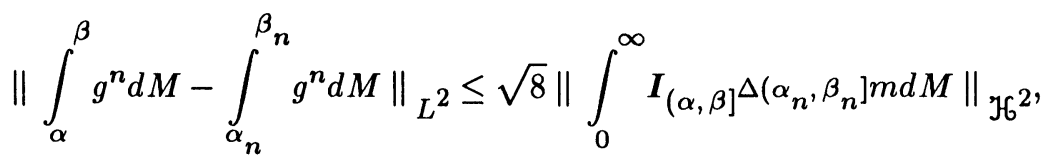

where $A \Delta B$ denotes the set $(A \backslash B) \cup(B \backslash A)$. Therefore,

$$
\begin{gathered}
\left\|x_{\beta}-x_{\alpha}-\int_{\alpha}^{\beta} g^{n} d M\right\|_{L^{2}} \\
\leq\left\|x_{\beta}-x_{\alpha}-\left(x_{\beta_{n}}-x_{\alpha_{n}}\right)\right\|_{L^{2}}+\left\|x_{\beta_{n}}-x_{\alpha_{n}}-\int_{\alpha_{n}}^{\beta_{n}} g^{n} d M\right\|_{L^{2}} \\
+\sqrt{8} \| \int_{0}^{\infty} I_{(\alpha, \beta] \Delta\left(\alpha_{n}, \beta_{n}\right]^{m d M} \|_{J_{6}{ }^{2}}}
\end{gathered}
$$


Since $\left(x_{t}\right)_{t \geq 0}$ and the stochastic integral are cádlág processes, $\alpha_{n} \rightarrow \alpha, \beta_{n} \rightarrow \beta$ as $n \rightarrow \infty$, we can select $n_{0}$ so great that first and third components are less than $\epsilon / 3$ for $n>n_{0}$.

Next we have

$$
\begin{aligned}
& \left\|x_{\beta_{n}}-x_{\alpha_{n}}-\int_{\alpha_{n}}^{\beta_{n}} g^{n} d M\right\|_{L^{2}} \\
& =\| \sum_{k=0}^{\infty} I_{B_{k}^{n} \backslash A_{k}^{n}\left[x_{(k+1) 2}-n-x_{k 2}-n-\int_{k 2}^{(k+1) 2-n} g^{n, k} d M\right] \|} L^{2} \\
& \leq \sum_{k=0}^{\infty} \epsilon /\left(3 \cdot 2^{k}\right)=\epsilon / 3 \text { for } n=1,2, \ldots .
\end{aligned}
$$

Since $\epsilon>0$ is arbitrary and fixed, we obtain

$$
\lim _{n \rightarrow \infty}\left\|\left(x_{\beta}-x_{\alpha}\right)-\int_{\alpha}^{\beta} g^{n} d M\right\|_{L^{2}}=0 .
$$

Theorem 1: Let $M$ be a semimartingale in $\mathfrak{H}^{2}$ and let $m$ be a process in $L(M) \cap X^{2}$. Suppose $\mathscr{R}_{0}$ is a predictable set-valued process integrably bounded by $m$. If $x=\left(x_{t}\right)_{t \geq 0}$ is a cádlág process such that $x_{t}-x_{s} \in c l{ }_{L} \int_{s}^{t} \Re d M$ a.s. for every stopping time $T$ and $s, t, T \leq s \leq t \leq \infty$, then for every $\epsilon>0$ there exists a process $H \in c l_{L(M)}{ }_{M}(\mathscr{R})$ such that

$$
\sup _{t \geq T}\left\|x_{t}-x_{T}-\int_{T}^{t} H d M\right\|_{L^{2}}<\epsilon .
$$

Proof: Let $\epsilon>0$ be fixed. By the Fundamental Theorem of Local Martingales and the Bichteler-Dellacherie Theorem $M$ has a decomposition $M=N+A$ such that the jumps of the local martingale $N$ are bounded by $\epsilon\left(3 C_{2}\|m\|_{S^{2}}\right)^{-1}$. Define recursively

$$
\begin{gathered}
T_{0}=T \\
T_{k+1}=\inf \left\{t \geq T_{k}:\left(\int_{T_{k-1}}^{t} d[N, N]\right)^{1 / 2}+\int_{T_{k-1}}^{t}|d A| \geq \epsilon\left(3 C_{2}\|m\|_{S^{2}}\right)^{-1}\right. \\
\text { or } \left.\left|x_{t}-x_{T_{k-1}}\right|>\epsilon / 3\right\} .
\end{gathered}
$$

Then $\left(T_{k}\right)$ increase to infinity a.s. [12, p. 192].

By Lemma 1 , for every $k=1,2, \ldots$, there exists a selector $H_{k} \in \varphi_{M}\left(\mathscr{B}_{0}\right)$ such that

$$
\left\|x_{T_{k}}-x_{T_{k-1}}-\int_{T_{k-1}}^{T_{k}} H_{k} d M\right\|_{L^{2}}<\frac{1}{2^{k}} \cdot \epsilon / 3 .
$$

Next, take any $H_{0} \in \varphi_{M}(\Re)$ and define $H=H_{0} \boldsymbol{I}_{[0, T)}+\sum_{k=1}^{\infty} H_{k} \boldsymbol{I}_{\left[T_{k-1}, T_{k}\right)}$. Let us claim that $H \in c l_{L(M)}{ }^{\varphi} M_{M}(\Re)$. Indeed, the set $c l_{L(M)}{ }^{\varphi} M_{M}(\Re)$ is closed in $L(M)$ and decomposable. 
Then $H_{n}=H_{0} \boldsymbol{I}_{[0, T)}+\sum_{k=1}^{n} H_{k} \boldsymbol{I}_{\left[T_{k-1}, T_{k}\right)}$ belongs to $\varphi_{M}\left(\Re_{0}\right)$. Since $H_{n}$ tends to $H$ for all $(t, \omega)$ and $\left|H_{n}\right|<m \in L(M)$ for each $n=1,2, \ldots$, then by the Lebesgue Dominated Convergence Theorem $H_{n}$ tends to $H$ in $L(M)$ [12].

Now we have

$$
\begin{gathered}
\sup _{t \geq T}\left\|x_{t}-x_{T}-\int_{T}^{t} H d M\right\|\left\|_{L^{2}}=\sup _{k \geq 1} \sup _{k-1} \sup _{t<T_{k}}\right\| x_{t}-x_{T}-\int_{T}^{t} H d M \|_{L^{2}} \\
\leq \sup _{k \geq 1} \sup _{k-1} \leq t<T_{k}\left\|x_{t}-x_{T_{k-1}}\right\| L_{L^{2}}+\sup _{k \geq 2}\left\|\sum_{i=1}^{k-1}\left(x_{T_{i}}-x_{T_{i-1}}-\int_{T_{i-1}} H d M\right)\right\|_{L^{2}} \\
+\sup _{k \geq 1} \sup _{k-1 \leq t<T_{k}}\left\|\int_{T_{k-1}}^{t} H d M\right\| L_{L^{2}}=I_{1}+I_{2}+I_{3} .
\end{gathered}
$$

By the definition of $T_{k}$ we obtain $\sup _{T_{k-1} \leq t<T_{k}}\left|x_{t}-x_{T_{k-1}}\right|<\epsilon / 3$ for $k=1,2, \ldots$, and a.e. $\omega \in \Omega$. Therefore, $I_{1}<\epsilon / 3$.

$$
\begin{gathered}
I_{2} \leq \sup _{k \geq 2} \sum_{i=1}^{k-1}\left\|x_{T_{i}}-x_{T_{i-1}}-\int_{T_{i-1}}^{T_{i}} H_{i} d M\right\|_{L^{2}} \\
\leq \sum_{i=1}^{\infty}\left\|x_{T_{i}}-x_{T_{i-1}}-\int_{T_{i-1}}^{T_{i}} H d M\right\|_{L^{2}} \leq \epsilon / 3 \sum_{i=1}^{\infty} \frac{1}{2^{i}}=\epsilon / 3 .
\end{gathered}
$$

Now let us observe that

$$
\begin{gathered}
\left\|\int_{T_{k-1}}^{t} H d M\right\|_{L^{2}} \leq\left\|H \cdot I_{\left(T_{k-1}, t\right]} \cdot M\right\|_{S^{2}} \leq C_{2}\left\|H \cdot I_{\left(T_{k-1}, t\right]} \cdot M\right\|_{J^{2}} \\
\leq C_{2}\|m\|_{S^{2}}\left\|\left(\int_{T_{k-1}}^{t} d[N, N]\right)^{1 / 2}+\int_{T_{k-1}}^{t}|d A|\right\|_{L^{2}}
\end{gathered}
$$

Therefore, by the definition of $\left(T_{k}\right)$ we get $I_{3} \leq \epsilon / 3$ and we are done.

Theorem 2: Let all assumptions of Theorem 1 be satisfied. If, moreover, $\mathbb{B}$ takes on convex values, then there exists a process $H \in c_{L(M)}{ }^{\varphi}{ }_{M}\left(\mathscr{R}_{0}\right)$ such that

$$
x_{t}=x_{T}+\int_{T}^{t} H d M \text { a.s. for each } t \geq T .
$$

Proof: By virtue of Theorem 1, there exists a sequence $\left(H^{n}\right)$ in $c l_{L(M)}{ }_{M}\left(\mathscr{R}_{0}\right)$ such that

$$
\sup _{t \geq T}\left\|x_{t}-x_{T}-\int_{T}^{t} H^{n} d M\right\|_{L^{2}} \rightarrow 0 \text { as } n \rightarrow \infty .
$$

We show that the set $\left(H^{n}\right)$ is weakly compact in $L(M)$. Since 


$$
\|H\|_{M} \leq\left(\int_{\Omega} \int H^{2} d[N, N] d P\right)^{1 / 2}+\left(\int_{\Omega}\left(\int|H||d A|\right)^{2}\right)^{1 / 2}
$$

then this norm is weaker from the norm defined by the sum of norms in $\ell^{2}\left(\Omega, \ell^{2}\left(\mathbb{R}_{+}, \mu\right)\right)$ and $\mathcal{L}^{2}\left(\Omega, \mathcal{L}^{1}\left(\mathbb{R}_{+}, \nu\right)\right)$, where $\mu$ and $\nu$ denote measures generated by $[N, N]$ and $|A|$ respectively. The set $\left(H^{n}\right)$ is integrably bounded, so it is weakly compact in the first space mentioned above by [4, Th. II.9]. It is also weakly compact in the second space, because the weak compactness of bounded sets in $\mathcal{L}^{2}(\Omega, E)$ and $\mathcal{L}^{1}(\Omega, E)$ is equivalent ([4]) and it follows by [9, Th. 2.1] that the set of selectors of integrable bounded set-valued functions is weakly compact in $\mathcal{L}^{1}\left(\Omega, \ell^{1}(\nu)\right)=$ $\mathcal{L}^{1}\left(\Omega \times \mathbb{R}_{+}, P \times \nu\right)$. Therefore, we deduce that $\left(H^{n}\right)$ has a weak cluster point $H$ in $c l_{L(M)} S_{M}\left(\mathscr{R}_{0}\right)$. On the other hand, $x_{t}-x_{T}$ and $\int_{T}^{t} H d M$ are weak cluster points of a weak convergent sequence $\int_{T}^{t} H^{n} k d M$ in $L^{2}\left(\mathscr{F}_{t}\right)$ for each $t \geq \stackrel{T}{T}$. Therefore $x_{t}-x_{T}$ is a modification of $\left(\int_{T}^{t} H d M\right)_{t} \geq T$. Then, by $[12$, I. Th. 2],

$$
x_{t}=x_{T}+\int_{T}^{t} H d M \text { a.s. for each } t \geq T
$$

\section{References}

[1] Ahmed, N.U., Existence of solutions of nonlinear stochastic differential inclusions on Banach spaces, In: Proc. of the First World Congress of Nonlinear Analysts, (ed. by V. Lakshmikantham), Walter de Gruyter, Berlin 1995, (in press).

[2] Aubin, J. and Cellina, A., Differential Inclusions, Springer-Verlag, Berlin, Heidelberg, New York 1984.

[3] Avgerinos, E.P. and Papageorgiou, N.S., Random nonlinear evolution inclusions in reflexive Banach spaces, Proc. of the AMS 104 (1988), 293-299.

[4] Bombal, F., On some subsets of $L_{1}(\mu, E)$, Czech. Math. J. 41 (1991), 170-178.

[5] Emery, M., Stabilité des solutions des équations différentialles stochastiques, $Z$. Wahrscheinlichkeitstheorie 41 (1978), 241-262.

[6] Hiai, F. and Umegaki, H., Integrals, conditional expectations, and martingales of multivalued functions, J. Multivar. Anal. 7 (1977), 149-182.

[7] Hiai, F., Multivalued stochastic integrals and stochastic differential inclusions, Division of Applied Math, Research Inst. of Applied Electricity, Sapporo 060, Japan (not published).

[8] Kisielewicz, M., Properties of solution set of stochastic inclusions, J. Appl. Math. Stoch. Anal. 6 (1993), 217-236.

[9] Kisielewicz, M., Differential Inclusions and Optimal Control, Kluwer Acad. Publ. and Polish Sci. Publ., Warszawa, Dordrecht, Boston, London 1991.

[10] Kravec, T.N., To the question on stochastic differential inclusions, Teoria Slucajnich. Processov (Theory of Random Processes) 15 (1987), 54-59 (in Russian).

[11] Motyl, J., On the solution of stochastic differential inclusion, J. Math. Anal. Appl. 191 (1995), (to appear).

[12] Protter, P., Stochastic Integration and Differential Equations (A New Approach), SpringerVerlag, Berlin, Heidelberg, New York 1990.

[13] Wu, R., Stochastic Differential Equations, Research Notes in Math. Series 1301985. 


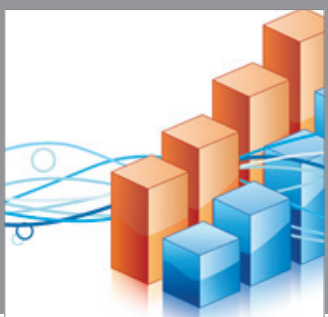

Advances in

Operations Research

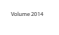

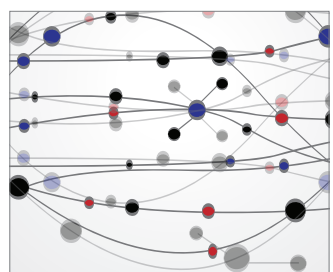

\section{The Scientific} World Journal
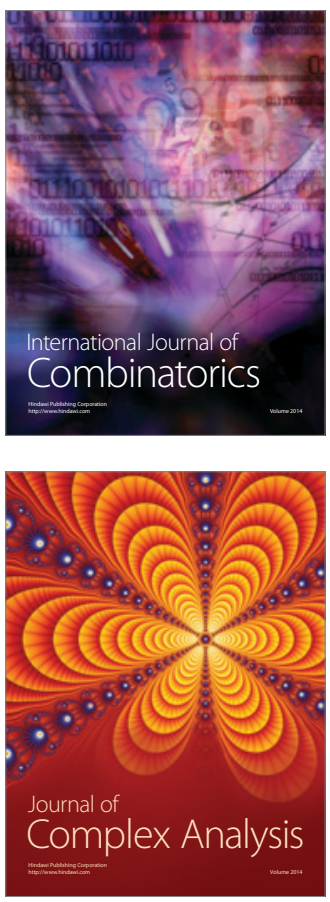

International Journal of

Mathematics and

Mathematical

Sciences
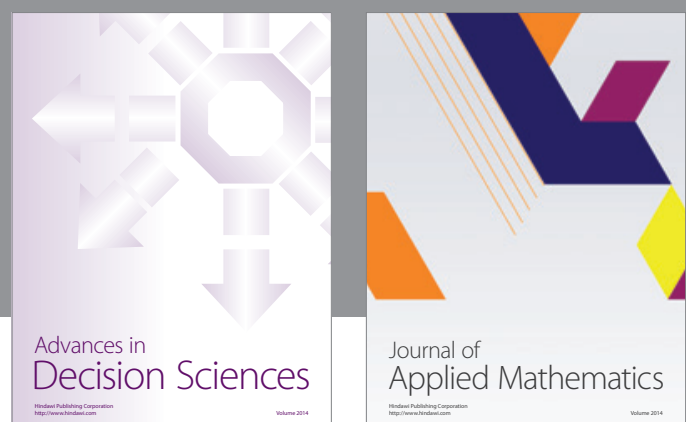

Journal of

Applied Mathematics
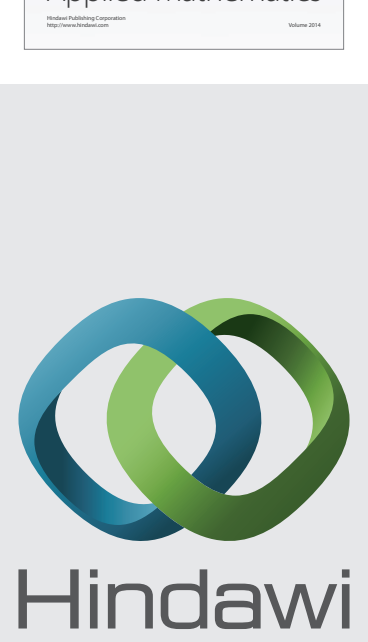

Submit your manuscripts at http://www.hindawi.com
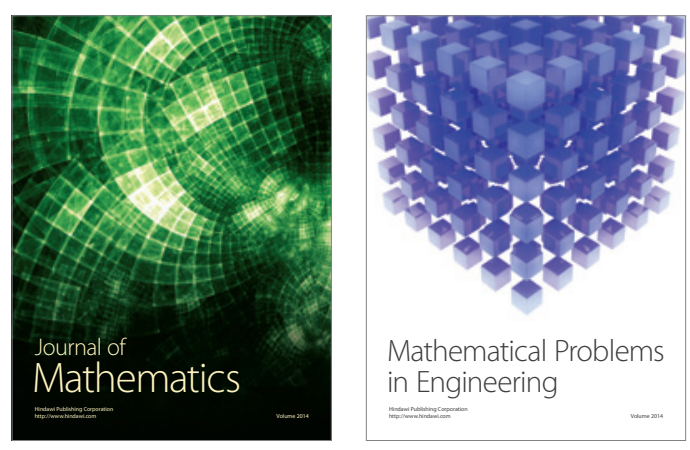

Mathematical Problems in Engineering
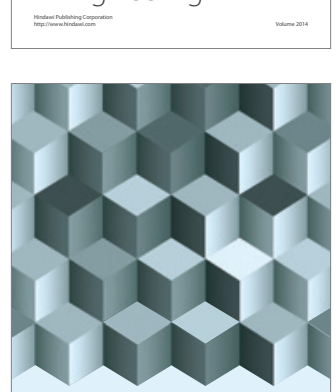

Journal of

Function Spaces
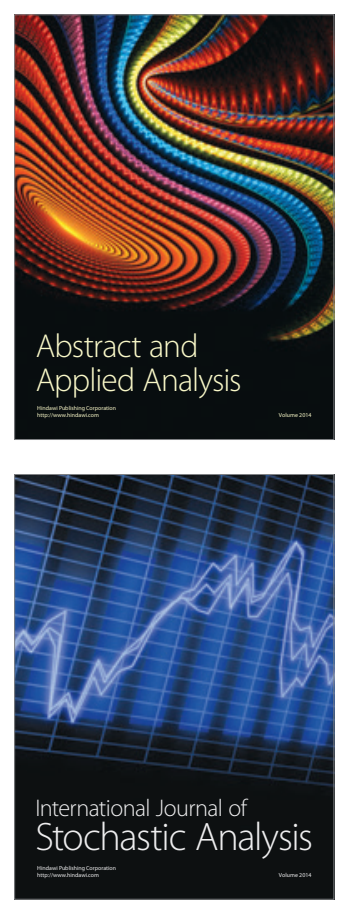

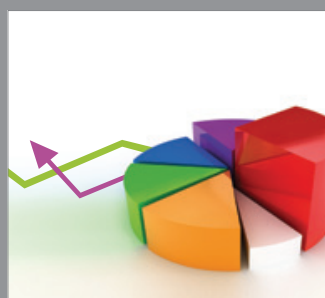

ournal of

Probability and Statistics

Promensencen
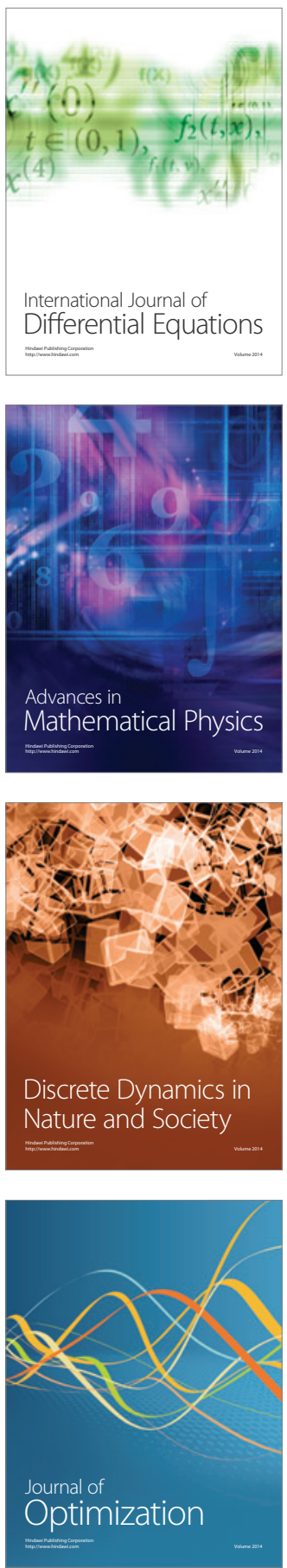